ARTICLE

\title{
Ultra-narrow metallic armchair graphene nanoribbons
}

Amina Kimouche ${ }^{1}$, Mikko M. Ervasti ${ }^{2}$, Robert Drost ${ }^{1}$, Simo Halonen ${ }^{3}$, Ari Harju ${ }^{2}$, Pekka M. Joensuu ${ }^{3}$, Jani Sainio ${ }^{1} \&$ Peter Liljeroth ${ }^{1}$

Graphene nanoribbons (GNRs)-narrow stripes of graphene-have emerged as promising building blocks for nanoelectronic devices. Recent advances in bottom-up synthesis have allowed production of atomically well-defined armchair GNRs with different widths and doping. While all experimentally studied GNRs have exhibited wide bandgaps, theory predicts that every third armchair GNR (widths of $N=3 m+2$, where $m$ is an integer) should be nearly metallic with a very small bandgap. Here, we synthesize the narrowest possible GNR belonging to this family (five carbon atoms wide, $N=5$ ). We study the evolution of the electronic bandgap and orbital structure of GNR segments as a function of their length using low-temperature scanning tunnelling microscopy and density-functional theory calculations. Already GNRs with lengths of $5 \mathrm{~nm}$ reach almost metallic behaviour with $\sim 100 \mathrm{meV}$ bandgap. Finally, we show that defects (kinks) in the GNRs do not strongly modify their electronic structure.

\footnotetext{
${ }^{1}$ Department of Applied Physics, Aalto University School of Science, PO Box 15100, Aalto 00076, Finland. ${ }^{2}$ COMP Centre of Excellence, Department of Applied Physics, Aalto University School of Science, PO Box 11100, Aalto 00076, Finland. ${ }^{3}$ Department of Chemistry, Aalto University School of Chemical Technology, PO Box 16100, Aalto 00076, Finland. Correspondence and requests for materials should be addressed to P.L. (email: peter.liljeroth@aalto.fi).
} 
G raphene nanoribbons (GNRs) are a new class of materials that have promising applications in next-generation nanoelectronic and optoelectronic devices ${ }^{1-3}$. These systems have been thoroughly studied theoretically at various levels of sophistication ${ }^{4-9}$. According to these studies, the electronic and magnetic properties can be tuned by the nanoribbon chemical structure and edge geometry, ranging from semiconductors ${ }^{7,8}$ to spin-polarized half-metals ${ }^{6}$. Zigzag edge structure is predicted to result in spin-polarized edge states, with potential applications in spintronics ${ }^{4,6,10}$. On the other hand, in armchair GNRs, quantum confinement opens a bandgap that sensitively depends on the ribbon width, allowing in principle for the design of GNR-based structures with tuneable properties. The armchair ribbons can be grouped into three families, that is, $N=3 p, N=3 p+1$ and $N=3 p+2$, where $p$ is an integer and $N$ the number of carbon atoms along the GNR width $^{4,11} . N=3 p$ and $N=3 p+1$ families have wide bandgaps that scale inversely with the ribbon width. In contrast, simple models predict the family $N=3 p+2$ to be metallic with zero bandgap $^{4,12}$. More sophisticated models taking into account, for example, edge relaxation predict non-vanishing but small bandgaps ${ }^{8,13}$. These narrow armchair GNRs with narrow or vanishing bandgaps would form ideal molecular wires to be used as interconnects in molecular scale circuitry. However, this requires atomic-level control of the edge structure, which is far beyond the existing top-down approaches such as electron beam lithography. Recently, tremendous progress has been made in bottom-up chemical synthesis of GNRs from molecular precursors ${ }^{14-23}$. The synthesis of these ribbons proceeds in two thermally activated steps ${ }^{14}$. First step is the cleavage of halogens from the precursors and the formation of a covalently coupled polymer through radical addition reaction. The linear chain is converted into graphene in the second step that involves cyclodehydrogenation at a higher temperature. By changing the monomer design, the fabrication of a wide range of GNRs including different widths and doping can be achieved.

Despite several kinds of armchair GNRs having been synthesized via specific molecular precursors ${ }^{2,16-19,21-26}$, the studied widths have been rather limited. Most of them belong to the wide bandgap $N=3 p+1$ family and narrow bandgap, nearmetallic behaviour has not been verified experimentally. We focus on the $N=3 p+2$ family and target the narrowest member of this family with $N=5$. The synthesis starts with a dibromoperylene molecule, which undergoes dehalogenation and cyclodehydrogenation steps to yield atomically perfect $N=5$ armchair GNRs. This width is predicted to be metallic within a nearest-neighbour tight-binding model ${ }^{4,7}$. More realistic calculations predict the presence of a bandgap, but it should remain much smaller than that found in armchair GNRs of the other families ${ }^{7}$. We confirm these theoretical predictions and measure experimentally a $\sim 100 \mathrm{meV}$ bandgap in long GNRs using low-temperature scanning tunnelling microscopy (STM). This near-metallic regime is already reached in GNRs of six perylene monomer units, that is, with lengths longer than $5 \mathrm{~nm}$. In addition, we study the effect of the ribbon length on the electronic structure and map out the local density of states (LDOS) in real space using scanning tunnelling spectroscopy. The experimental results are corroborated by density-functional theory (DFT) calculations that are used to identify the fingerprints of molecular orbitals as a function of the GNR length. Finally, we show that defects (kinks) in the GNRs do not destroy the near-metallic behaviour. Our results demonstrate that this armchair GNR subfamily can be used as molecular wires that should exhibit metallic behaviour at room temperature. This suggest that these GNRs would form ideal interconnects in molecular scale electronic circuitry.

\section{Results}

Growth of $N=\mathbf{5}$ armchair GNRs. We grew armchair GNRs in ultrahigh vacuum (UHV) using the on-surface polymerization with dibromoperylene $\mathrm{C}_{20} \mathrm{H}_{10} \mathrm{Br}_{2}$ (DBP) as the molecular precursor (Fig. 1a) ${ }^{23}$. This process yields very narrow 5 -GNRs with armchair edge structure. Details of the sample preparation can be found in the Methods section. The resulting GNRs were characterized by low-temperature STM at $T=5 \mathrm{~K}$. We used a mixture of precursor monomers with either parallel or antiparallel positions of $\mathrm{Br}$ atoms (3,9-DBP or 3,10-DBP). Regardless of the positions of the $\mathrm{Br}$ atoms, fully conjugated ribbons are formed. A typical overview STM scan is shown in Fig. 1b. These ribbons align and assemble along or perpendicular to the direction of the herringbone reconstruction of the $\mathrm{Au}(111)$ substrate. Most ribbons are isolated and easily manipulated along the ribbon axis due to the weak interaction with the underlying $\mathrm{Au}(111)$ substrate. While most of the GNRs are straight, there is a significant number of non-straight GNRs. These kinked ribbons are formed by two (or more) straight segments connecting during the initial polymerization step with an angle of $30^{\circ}$. The structure and electronic properties of these kinked ribbons will be discussed in detail later. Statistics on the ribbon lengths and the fraction of kinked ribbons are given in Supplementary Fig. 1. Figure 1c shows a high-resolution STM image with GNRs with several different lengths. The shortest ribbons we observe consist of two monomers, a medium length ribbon of 5-monomer units is highlighted with an overlaid model structure. We can easily determine the number of monomer units by counting the number of benzene rings (lobes) along the armchair edges of the GNRs.

Evolution of GNR orbitals as a function of length. We have performed detailed differential conductance $(\mathrm{d} I / \mathrm{d} V)$ spectroscopy

a

a

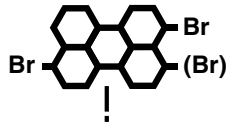

Polymerization at $200{ }^{\circ} \mathrm{C}$ i

Cyclodehydrogenation at $320^{\circ} \mathrm{C}$
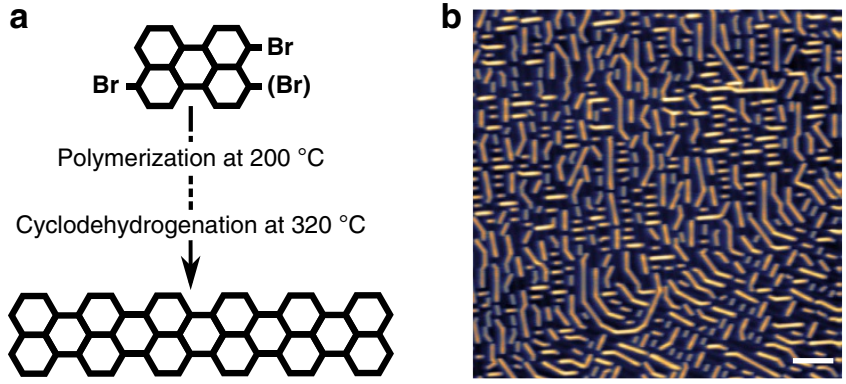

c

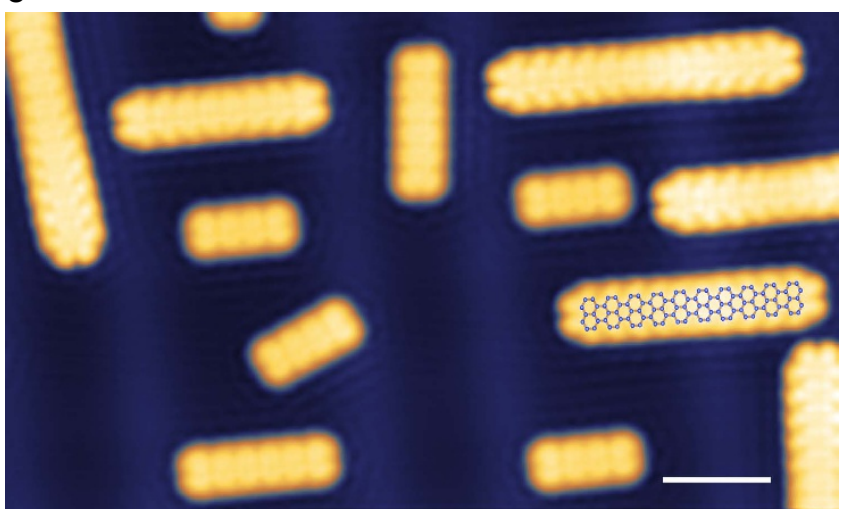

Figure 1 | Bottom-up synthesis of $\mathbf{N}=\mathbf{5}$ armchair GNRs. (a) Reaction scheme of the polymerization of the DBP precursor to atomically well defined $N=5$ armchair GNRs. (b) Overview STM image after cyclodehydrogenation at $320^{\circ} \mathrm{C}$, showing straight and kinked GNRs $(V=500 \mathrm{mV}, I=50 \mathrm{pA}$; scale bar, $10 \mathrm{~nm}$ ). (c) Zoomed-in STM topography of different ribbon lengths $(V=300 \mathrm{mV}, I=50 \mathrm{pA}$; scale bar, $2 \mathrm{~nm}$ ). 
experiments as a function of the ribbon length to probe the corresponding changes in their electronic structure. In the following, we will use two ribbon lengths (3 and 5 monomer units) to highlight how the ribbon electronic states change with length. A characteristic spectrum (red curve, Fig. 2a) recorded close to the edge of a 3-monomer GNR exhibits a pronounced shoulder at $-1.02 \mathrm{~V}$ and a prominent peak at $-160 \mathrm{mV}$ (labelled states 1 and 2, respectively). These states correspond to the highest occupied molecular orbitals HOMO-1 (state 1) and HOMO (state 2). At positive bias, we probe the lowest unoccupied states. We find states at $540 \mathrm{mV}$ (state 3) and $1.8 \mathrm{~V}$ (state 4), which correspond to the lowest and second lowest unoccupied molecular orbitals (LUMO and LUMO +1 ). The additional feature observed in the $\mathrm{d} I / \mathrm{d} V$ spectrum around $1.2 \mathrm{~V}$ did not show any contrast in the $\mathrm{d} I / \mathrm{d} V$ map and it originates from the background signal (black line).

We probe the symmetries of the different molecular orbitals by mapping the spatially resolved $\mathrm{d} I / \mathrm{d} V$ signal at the energies corresponding to the resonances (Fig. 2c). The experimental maps show that both the HOMO and LUMO are extended through the ribbon, in contrast to the localized end states observed on wider armchair GNRs ${ }^{2,19}$. The LUMO +1 has one more nodal plane compared with the LUMO as expected. The corresponding calculated LDOS maps (Fig. 2e) are in excellent agreement with the experimental results, clearly reproducing the occupied and unoccupied states. The unoccupied orbitals yield broader resonances in the $\mathrm{d} I / \mathrm{d} V$ spectra and their spatial maps are not as well resolved as for the occupied states. This is likely to be caused by shorter lifetime of the tunnelling electrons on these states leading to increased lifetime broadening. Nevertheless, the orbitals can be identified by the conductance mapping.

Figure $2 \mathrm{~b}$ shows the $\mathrm{d} I / \mathrm{d} V$ spectra acquired at different locations along a 5-monomer GNR. The corresponding experimental and calculated conductance maps are shown in Fig. 2d,f. The occupied states are found at -550 and $26 \mathrm{mV}$ referred as HOMO- 1 and HOMO, respectively. The orbital corresponding to the HOMO of an isolated ribbon is found at positive bias, that is, the ribbon has become positively charged on the $\mathrm{Au}(111)$ substrate. We find this transition to occur between 4- and 5-monomer long ribbons. It is consistent with the known p-doping of bulk graphene on a gold substrate 27,28 . The unoccupied states found at $250 \mathrm{mV}$, $850 \mathrm{mV}, 1.25 \mathrm{~V}$ and $1.57 \mathrm{~V}$ are referred to as LUMO, LUMO + 1, $\mathrm{LUMO}+2$ and LUMO +3 , respectively. These unoccupied states follow the energies expected for one-dimensional particle-in-a-box states of massless Dirac fermions. In addition to these resonances corresponding to single molecular orbitals, there are two further peaks (peaks 7 and 8 in Fig. 2b) in the experimental $d I / d V$ spectra. According to the DFT calculations, these correspond to several closely spaced orbitals (Supplementary Fig. 2). This is further corroborated by simulated LDOS maps that nicely reproduce the spatial features of the experimental maps.

In addition to quantum confinement, which makes the electronic structure of finite graphene ribbons very sensitive to the width, finite-size effects have to be taken into account to determine the scaling of the HOMO-LUMO energy gap with the length of the ribbon. Here, we will examine how the finite-size effects affect the energies of molecular levels in the transition from short to long ribbons. Experimental orbital energies with respect to Fermi level extracted from $\mathrm{d} I / \mathrm{d} V$ spectra are depicted in Fig. 3a for different ribbon lengths. As can be seen, the gap between the highest occupied and the lowest unoccupied molecular orbitals closes quickly with increasing ribbon length. As indicated earlier, the HOMO level crosses the Fermi level between 4 - and 5-monomer long ribbons, that is, the ribbon becomes positively charged (this is illustrated in Supplementary Fig. 3). The middle of the gap extrapolates to $0.20 \pm 0.02 \mathrm{eV}$ a

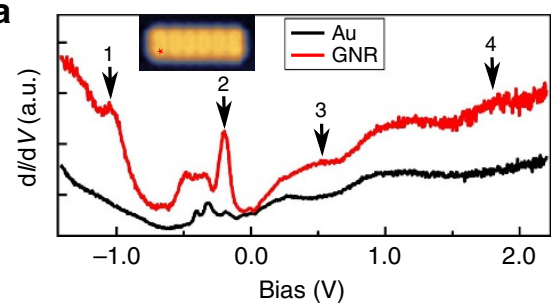

C

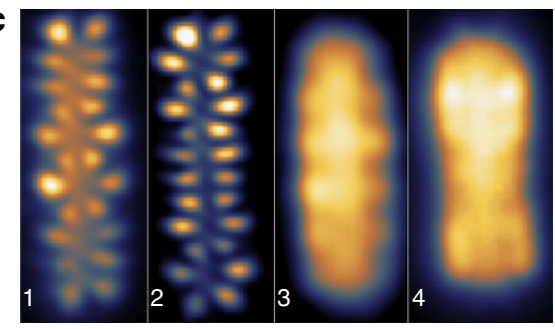

e

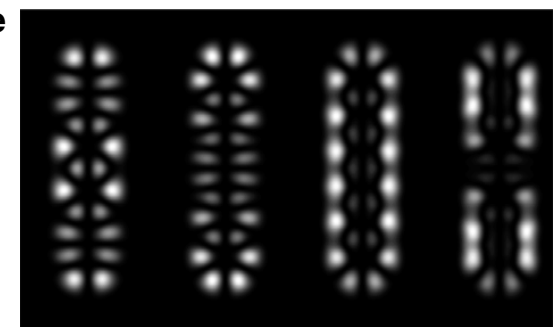

b
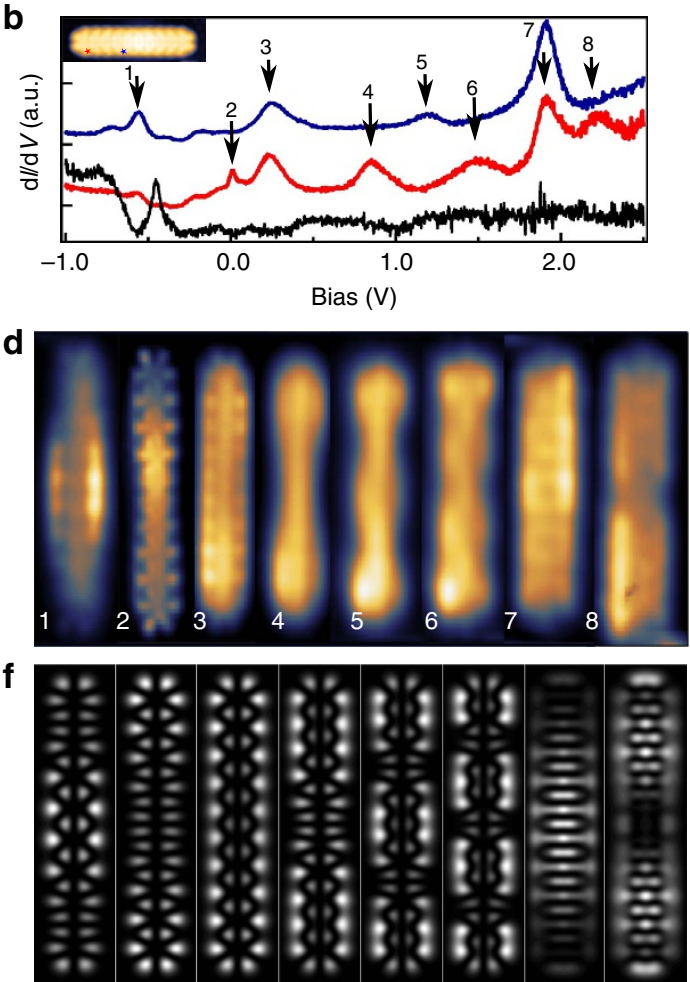

Figure $\mathbf{2} \mid \mathbf{d} \mathbf{l} / \mathbf{d} \boldsymbol{V}$ spectroscopy and real-space imaging of the GNR wavefunctions. (a,b) $d / / d V$ spectra acquired on three (a) and five (b) monomer GNR with a metallic tip. Location of the spectra are marked in the STM topographies in the insets and the black curve is measured on Au(111). The red and blue curves are shifted for clarity. (c-f) Experimental constant-height $\mathrm{d} / / \mathrm{dV}$ maps $(\mathbf{c}, \mathbf{d})$ and the corresponding calculated LDOS maps (e,f) for three (c,e) and five $(\mathbf{d}, \mathbf{f})$ monomer GNRs at bias voltages corresponding to the positions marked with arrows in $\mathbf{a}, \mathbf{b}$. 
versus gold Fermi level, in agreement with the doping of bulk graphene on $\mathrm{Au}(111)^{27,28}$. There is no significant abrupt shift of the orbital energies due to the charging of the ribbons, which indicates that the charging energy is small and can be neglected ${ }^{29}$. This also implies that the STM transport gap is nearly equal to the single-particle HOMO-LUMO gap.

We plot the HOMO-LUMO gap as a function of the length of the ribbon in Fig. $3 \mathrm{~b}$ along with values from the DFT calculations on isolated ribbons. The HOMO-LUMO gap decreases very quickly with increasing ribbon length (scaling roughly as $1 / L^{2}$ ) and is below $200 \mathrm{meV}$ already for 5-monomer ribbons (length of ca. $4 \mathrm{~nm}$ ). It saturates to value of $0.10 \pm 0.02 \mathrm{eV}$ corresponding to a small but finite energy gap in long ribbons. The spacing between the HOMO and HOMO-1 also decreases, but with a different scaling (roughly $1 / L$ ). This scaling indicates that these states can be viewed as quantum confined levels of particles with linear dispersion as expected in graphene. The HOMO-1-HOMO gap extrapolates to 0 as one would expect for the level spacing within a subband in infinite ribbons. These experimental observations are well reproduced by DFT calculations.

Detailed comparison between theory and experiment shows that DFT overestimates the experimental HOMO-LUMO gap. This is likely to be caused by the omission of the substrate, the resulting charging of the ribbon and the strong metallic screening. Furthermore, the neutral isolated ribbons are predicted to have singlet edge states for lengths $\geq 7$ monomer units (Supplementary Fig. 4 and Supplementary Methods). The narrow bandgap predicted earlier by $\mathrm{DFT}^{7}$ matches very well with our measured and calculated gaps. These values differ from those reported recently by Zhang et al. ${ }^{18}$, who obtained a bandgap of $2.8 \mathrm{eV}$. They used another precursor molecule (tetrabromonaphthalene), which also results in $N=5$ armchair GNRs. Comparison with our results suggests that they have

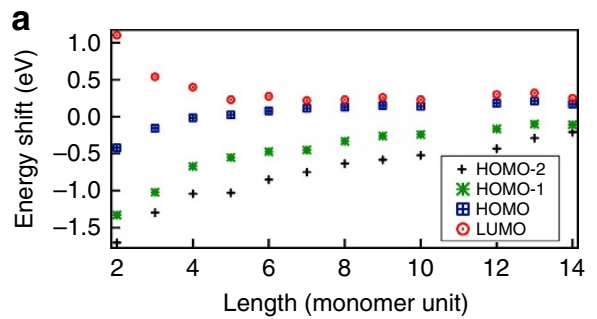

identified the most prominent features in the spectra as the conduction and valence band edges (see for example the peak at around $2.0 \mathrm{~V}$ in Fig. 2b). However, our experiments show that there are other, lower lying orbitals and the actual HOMO-LUMO gap on $\mathrm{Au}(111)$ reaches value of $\sim 100 \mathrm{meV}$ in long ribbons. Finally, the many-body phenomena predicted for wider armchair ribbons ${ }^{30,31}$ or for zigzag ribbons ${ }^{32-34}$ are not expected for such a short segment of zigzag edge.

Kinked GNRs formed by connected straight segments. In addition to straight GNRs, also kinked ribbons are formed (see Fig. 1a). These ribbons typically consist of two straight segments connected at an angle of $150^{\circ}$ corresponding to a rotation of $30^{\circ}$ (Fig. 4a). Grain boundaries in graphene are known to accommodate carbon pentagon and heptagon defects ${ }^{35,36}$. Considering how two nanoribbons might connect, the creation of a pentagon does not require adding or removing carbon atoms from two straight ribbons. In addition, DFT calculations on GNRs with pentagon defects reproduce the experimental results better than structures with heptagons (see below and Supplementary Fig. 5).

We have measured $\mathrm{d} I / \mathrm{d} V$ spectra as well conductance maps on kinked ribbons and Fig. 4 shows a ribbon where 4-monomer and 5 -monomer long straight segments are joined. Figure $4 \mathrm{~b}$ shows a $\mathrm{d} I / \mathrm{d} V$ spectrum acquired at the kink exhibiting peaks at -120 and $100 \mathrm{mV}$ and a pronounced shoulder at $300 \mathrm{mV}$. These energies correspond to the HOMO - 1, HOMO and LUMO, respectively. Simulated $\mathrm{d} I / \mathrm{d} V$ maps for a pentagon connection (HOMO - 1, HOMO and LUMO shown in Fig. 4d) match the experimental results reasonably. The energy gap of the kinked ribbon is ca. $200 \mathrm{meV}$, which is close to a straight 9-monomer ribbon and smaller than expected for four- or five-monomer long GNRs. In addition, the molecular orbitals remain delocalized over

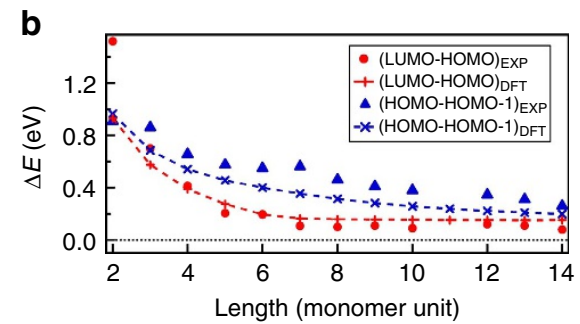

Figure 3 | Energies of molecular orbitals as function of ribbon length. (a) Energies of the different molecular orbitals (HOMO-2 to LUMO) as a function of the ribbon length. (b) Comparison between experimental and calculated energy gaps as a function of the ribbon length.
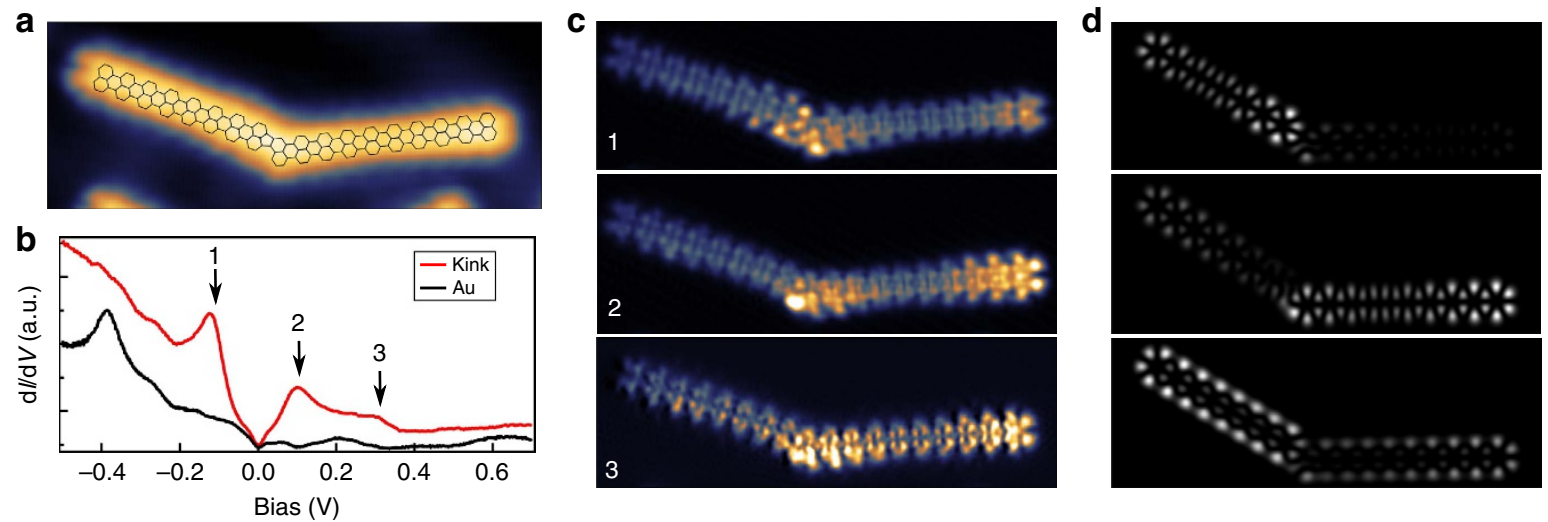

Figure 4 | Electronic structure of a kinked GNR with four- and five-monomer segments. (a) STM topography with an overlaid model shows the connection on the kink by a pentagon. (b) $\mathrm{d} l / \mathrm{d} V$ spectrum acquired at the kink (red line), an Au(111) spectrum (black line) is provided for comparison. (c) Constant-height $\mathrm{d} l / \mathrm{d} V$ maps at energies labelled by arrows in $\mathbf{b}$. (d) Corresponding simulated LDOS maps. 
the whole ribbon and hence, the kinks would not be expected to strongly affect the GNR conductivity.

\section{Discussion}

Our results demonstrate nearly metallic behaviour in ultranarrow, atomically well-defined graphene nanoribbons. We have synthesized the narrowest member $(N=5)$ of the $N=3 p+2$ family that has been predicted to exhibit narrow bandgap. We have confirmed this prediction and studied the evolution of the electronic states as a function of the ribbon length in detail using low-temperature STM and numerical methods. A bandgap of $\sim 100 \mathrm{meV}$ is reached already in short ribbons of only $5 \mathrm{~nm}$ in length. In addition, kinked GNRs formed by connected straight armchair GNR segments also exhibit narrow gaps and delocalized electronic orbitals.

These experimental results suggest that this armchair GNR subfamily can be used as molecular wires, which should exhibit metallic behaviour at room temperature. This opens up new opportunities to connect different functional molecules in molecular electronic devices. In addition, it could be possible to integrate these nanoribbons with existing technology, where they have been suggested to outperform the present $\mathrm{Cu}$ interconnects both in terms of conductance properties and increased resistance to electromigration ${ }^{37-41}$.

\section{Methods}

Sample preparation. Experiments were conducted under UHV conditions. The substrate was a thin $\mathrm{Au}(111)$ film deposited on a (111)-terminated Ir single crystal. The Ir crystal is cleaned by repeated sputtering/annealing cycles at high temperature. Gold (Goodfellow, 99.995\%) was deposited from a high-temperature effusion cell at $1,180^{\circ} \mathrm{C}$. The deposition time was $2 \mathrm{~h}$ and the sample was kept at $310^{\circ} \mathrm{C}$ during the deposition. Growth of GNRs was carried out in a two-step process in which the molecular precursor, dibromoperylene $\mathrm{C}_{20} \mathrm{H}_{10} \mathrm{Br}_{2}$ (see Supplementary Methods for synthesis information) is first thermally sublimed onto the sample. During the first thermal activation step at $200{ }^{\circ} \mathrm{C}$, the biradical species produced after detachment of bromine diffuse along the surface to form a linear polymer chain. Subsequent annealing of the sample at $320^{\circ} \mathrm{C}$ leads to dehydrogenation resulting in the formation of $\mathrm{C}-\mathrm{C}$ bonds and fully conjugated GNRs with a well-defined width.

After the growth, the sample was inserted into a low-temperature STM operated at $5 \mathrm{~K}$ (Unisoku USM-1300), housed within the same UHV system. A Pt-Ir tip was used for topographic and spectroscopic measurements. Scanning tunnelling spectroscopy measurements were performed by lock-in amplifier (frequency $=719 \mathrm{~Hz}$, amplitude $=15 \mathrm{mV}$ (r.m.s.)) under open feedback.

DFT calculations. First-principles calculations of $N=5$ armchair GNR were performed using DFT as implemented in the FHI-aims package ${ }^{42}$. The default tight basis sets were used together with the Perdew-Burke-Ernzerhof exchangecorrelation functional in a spin-polarized calculation. The structure was fully relaxed until the force on each atom was smaller than $0.001 \mathrm{eV}^{-1}$ and total energies were converged to $10^{-6} \mathrm{eV}$. In the LDOS simulations, a Lorentzian broadening of $0.03 \mathrm{eV}$ of all the orbitals was used. Further details can be found in the Supplementary Information.

\section{References}

1. Li, X. L., Wang, X. R., Zhang, L., Lee, S. W. \& Dai, H. J. Chemically derived, ultrasmooth graphene nanoribbon semiconductors. Science 319, 1229-1232 (2008).

2. Koch, M., Ample, F., Joachim, C. \& Grill, L. Voltage-dependent conductance of a single graphene nanoribbon. Nat. Nanotechnol. 7, 713-717 (2012).

3. Silveiro, I., Ortega, J. M. P. \& de Abajo, F. G. Quantum nonlocal effects in individual and interacting graphene nanoribbons. Light. Sci. Appl. 4, e241 (2015).

4. Nakada, K., Fujita, M., Dresselhaus, G. \& Dresselhaus, M. S. Edge state in graphene ribbons: Nanometer size effect and edge shape dependence. Phys. Rev. B 54, 17954-17961 (1996).

5. Wakabayashi, K., Fujita, M., Ajiki, H. \& Sigrist, M. Electronic and magnetic properties of nanographite ribbons. Phys. Rev. B 59, 8271-8282 (1999).

6. Son, Y.-W., Cohen, M. L. \& Louie, S. G. Half-metallic graphene nanoribbons. Nature 444, 347-349 (2006).

7. Son, Y.-W., Cohen, M. L. \& Louie, S. G. Energy gaps in graphene nanoribbons. Phys. Rev. Lett. 97, 216803 (2006).
8. Barone, V., Hod, O. \& Scuseria, G. E. Electronic structure and stability of semiconducting graphene nanoribbons. Nano Lett. 6, 2748-2754 (2006).

9. Yazyev, O. V. A guide to the design of electronic properties of graphene nanoribbons. Acc. Chem. Res. 46, 2319-2328 (2013).

10. Yazyev, O. V. Emergence of magnetism in graphene materials and nanostructures. Rep. Prog. Phys. 73, 056501 (2010).

11. Yang, L., Park, C.-H., Son, Y.-W., Cohen, M. L. \& Louie, S. G. Quasiparticle energies and band gaps in graphene nanoribbons. Phys. Rev. Lett. 99, 186801 (2007).

12. Brey, L. \& Fertig, H. A. Electronic states of graphene nanoribbons studied with the Dirac equation. Phys. Rev. B 73, 235411 (2006).

13. Ezawa, M. Peculiar width dependence of the electronic properties of carbon nanoribbons. Phys. Rev. B 73, 045432 (2006).

14. Cai, J. et al. Atomically precise bottom-up fabrication of graphene nanoribbons. Nature 466, 470-473 (2010).

15. Zhang, X. et al. Experimentally engineering the edge termination of graphene nanoribbons. ACS Nano 7, 198-202 (2013).

16. Blankenburg, S. et al. Intraribbon heterojunction formation in ultranarrow graphene nanoribbons. ACS Nano 6, 2020-2025 (2012).

17. Bronner, C. et al. Aligning the band gap of graphene nanoribbons by monomer doping. Angew. Chem. Int. Ed. 52, 4422-4425 (2013).

18. Chen, Y.-C. et al. Tuning the band gap of graphene nanoribbons synthesized from molecular precursors. ACS Nano 7, 6123-6128 (2013).

19. van der Lit, J. et al. Suppression of electron-vibron coupling in graphene nanoribbons contacted via a single atom. Nat. Commun. 4, 2023 (2013).

20. Cai, J. et al. Graphene nanoribbon heterojunctions. Nat. Nanotechnol. 9, 896-900 (2014).

21. Chen, Y.-C. et al. Molecular bandgap engineering of bottom-up synthesized graphene nanoribbon heterojunctions. Nat. Nanotechnol. 10, 156-160 (2015).

22. Zhang, H. et al. On-surface synthesis of rylene-type graphene nanoribbons. J. Am. Chem. Soc. 137, 4022-4025 (2015).

23. Sakaguchi, H. et al. Width-controlled sub-nanometer graphene nanoribbon films synthesized by radical-polymerized chemical vapor deposition. Adv. Mater. 26, 4134-4138 (2014).

24. Linden, S. et al. Electronic structure of spatially aligned graphene nanoribbons on $\mathrm{Au}(788)$. Phys. Rev. Lett. 108, 216801 (2012).

25. Söde, H. et al. Electronic band dispersion of graphene nanoribbons via Fourier-transformed scanning tunneling spectroscopy. Phys. Rev. B 91, 045429 (2015).

26. Talirz, L. et al. Termini of bottom-up fabricated graphene nanoribbons. J. Am. Chem. Soc. 135, 2060-2063 (2013).

27. Joseph, M. W. et al. Extraordinary epitaxial alignment of graphene islands on Au(111). New J. Phys. 14, 053008 (2012).

28. Leicht, P. et al. In situ fabrication of quasi-free-standing epitaxial graphene nanoflakes on gold. ACS Nano 8, 3735-3742 (2014).

29. Swart, I., Gross, L. \& Liljeroth, P. Single-molecule chemistry and physics explored by low-temperature scanning probe microscopy. Chem. Commun. 47, 9011-9023 (2011).

30. Ijäs, M. et al. Electronic states in finite graphene nanoribbons: Effect of charging and defects. Phys. Rev. B 88, 075429 (2013).

31. Golor, M., Koop, C., Lang, T. C., Wessel, S. \& Schmidt, M. J. Magnetic correlations in short and narrow graphene armchair nanoribbons. Phys. Rev. Lett. 111, 085504 (2013).

32. Rivero, P., Jiménez-Hoyos, C. A. \& Scuseria, G. E. Entanglement and polyradical character of polycyclic aromatic hydrocarbons predicted by projected Hartree-Fock theory. J. Phys. Chem. B 117, 12750-12758 (2013).

33. Mizukami, W., Kurashige, Y. \& Yanai, T. More $\pi$ electrons make a difference: Emergence of many radicals on graphene nanoribbons studied by ab initio DMRG theory. J. Chem. Theory Comput. 9, 401-407 (2013).

34. Wu, C.-S. \& Chai, J.-D. Electronic properties of zigzag graphene nanoribbons studied by TAO-DFT. J. Chem. Theory Comput. 11, 2003-2011 (2015).

35. Terrones, H. \& Mackay, A. L. The geometry of hypothetical curved graphite structures. Carbon 30, 1251-1260 (1992).

36. Huang, P. Y. et al. Grains and grain boundaries in single-layer graphene atomic patchwork quilts. Nature 469, 389-392 (2011).

37. Naeemi, A. \& Meindl, J. D. Conductance modeling for graphene nanoribbon (GNR) interconnects. IEEE Electron Device Lett. 28, 428-431 (2007).

38. Murali, R., Yang, Y., Brenner, K., Beck, T. \& Meindl, J. D. Breakdown current density of graphene nanoribbons. Appl. Phys. Lett. 94, 243114 (2009).

39. Murali, R., Brenner, K., Yinxiao, Y., Beck, T. \& Meindl, J. D. Resistivity of graphene nanoribbon interconnects. IEEE Electron Device Lett. 30, 611-613 (2009).

40. Rakheja, S., Kumar, V. \& Naeemi, A. Evaluation of the potential performance of graphene nanoribbons as on-chip interconnects. Proc. IEEE 101, 1740-1765 (2013).

41. Schwierz, F. Graphene transistors. Nat. Nanotechnol. 5, 487-496 (2010). 
42. Blum, V. et al. Ab initio molecular simulations with numeric atom-centered orbitals. Comp. Phys. Commun. 180, 2175-2196 (2009).

\section{Acknowledgements}

This research made use of the Aalto Nanomicroscopy Center (Aalto NMC) facilities and was supported by the European Research Council (ERC-2011-StG no. 278698 PRECISENANO) and the Academy of Finland through its Centres of Excellence Program (projects no. 250280 and 251748). M.M.E. acknowledges financial support from the Finnish Cultural Foundation. We acknowledge the computational resources provided by Aalto Science-IT project and Finland's IT Center for Science (CSC).

\section{Author contributions}

A.K., R.D., J.S. and P.L. designed the experiments and A.K., R.D. and P.L. carried them out. M.M.E. and A.H. carried out DFT calculations. S.H. and P.M.J. synthesized the precursor molecules. A.K. and P.L. wrote the paper. All authors discussed and commented the paper.

\section{Additional information}

Supplementary Information accompanies this paper at http://www.nature.com/ naturecommunications

Competing financial interests: The authors declare no competing financial interests.

Reprints and permission information is available online at http://npg.nature.com/ reprintsandpermissions/

How to cite this article: Kimouche, A. et al. Ultra-narrow metallic armchair graphene nanoribbons. Nat. Commun. 6:10177 doi: 10.1038/ncomms10177 (2015).

(c) (i) This work is licensed under a Creative Commons Attribution 4.0 International License. The images or other third party material in this article are included in the article's Creative Commons license, unless indicated otherwise in the credit line; if the material is not included under the Creative Commons license, users will need to obtain permission from the license holder to reproduce the material. To view a copy of this license, visit http://creativecommons.org/licenses/by/4.0/ 